\title{
NETNEWS/USENET Newsgroup for Powder Diffractionists
}

It has been stated by many individuals that the major effect of computers on mankind will not be the increased ability to handle enormous calculations but rather to revolutionize our means of communication. The last five years have seen huge advances in the intercommunication between computers, and with the Internet worldwide network now enjoying greater use, individuals are in almost instant communication with colleagues half a world away. Whether we like it or not, the age of computer communication is now upon us.

Of particular interest to powder diffractionists is the formation of a crystallographic newsgroup that is accessible through local protocols which access the world-wide network and the USENET network, which is a world-wide conferencing system. Although this newsgroup was only formed in October, it is already very popular as a discussion forum for problems and ideas. The newsgroup is identified as sci.techniques.xtallography, and messages are easily entered into the system for all interested readers to review and make responses. Conversations may be carried on about theoretical or practical topics. The important feature is that the response that is the most help may come from someone that you would not have considered asking. The potential is limitless. With normal e-mail, your message must be directed at a specific recipient; but when using the newsgroup, you have the opportunity to query everyone in the group. Once the newsgroup message has been transmitted and received, the responses may either continue as newsgroup messsages or by direct e-mail, depending on whether the subject is of general interest or of interest only to the original message sender.

Some of the topics which were considered when the newsgroup was formed include: powder diffraction (X-ray and neutron); electron diffraction, single-crystal diffraction; Rietveld refinement; software; databases; data formats; hardware/equipment-alignment and calibration, trade or sale of old apparatus, source of specialized equipment; phase identification and quantification; sample preparation techniques; sources of materials and compounds; announcements; pre-review of articles; accuracy and validity of results; and positions wanted or available. Obviously, the list is endless. There are some things for which a newsgroup is not an appropriate forum. It is not an alternative to publication of structures or articles to bypass the peer review procedures. Nor should it be a forum to expound on philosophy rather than science.

One question with this type of information transfer is how broad or narrow the subject matter should be. It is possible to create a newsgroup on any scientific topiceven subsets of crystallography if enough user support is present. If a newsgroup covers a very broad range of topics, it may be too diverse for the requirements and interests of the more specialized participants. There could also be too many messages to conveniently read on a regular basis, although most good "newsreading" software allows the option of only browsing through article titles, without having to read every message. On the other hand, if the subject matter is narrowly focused and too specific, there may not be a "critical mass" of participants; and, thus, the newsgroup may be underutilized. Finding the optimum design will take time and discussion from people interested in the potential of this technology being applied to scientific communication.

Initiating access to the newsgroup requires only access to the network and local support for preparing and sending messages and receiving the messages. Normally this access requires a "news reader" program on the users local system. If you have not already been informed of its existence and you want to find out how to participate in this newsgroup, contact either your librarian, systems manager, or Lachlan Cranswick [E-mail:lachlan @dmp.csiro.au, fax: 61-3-646-3223] at CSIRO in Melbourne, Australia. An archive of messages from the newsgroup files may also be obtained by anonymous FTP at sol.dmp.csiro.au in the /pub/xtallography directory. These archive files are in an ascii "e-mail" format that can be read by any text editor, but preferably by an e-mail program that sees each "article" as a separate e-mail message.

In addition to newsgroups, there are other computer information centers available to crystallographers. Also in existence is the crystallography section of the European CONCISE bulletin board (telnet concise.level-7.co.uk; username:concise, password:concise, crystallography information in section /sigs/crystal) managed by Howard Flack (flack@ scsun.unige.ch) at the University of Geneva, Switzerland, which is available on a world-wide basis via telnet, e-mail, and ftp. The tables of contents of the IUCr journals are posted on this bulletin board. In addition, using either telnet, e-mail or FTP, you may obtain address lists for crystallographers and announcements from the American Crystallographic Association. The most recent ACA Newsletter contains the necessary information.

Computer users will welcome and rapidly adapt to newsgroups. For those of us not brought up with the ideas that computers are indispensable, it will take somewhat longer to adapt to computer communication. For those individuals who consider the computer as an intrusion into their orderly world, it may take considerable effort to initiate regular use of newsgroups. Regardless, the age of computer communication is here. Newsgroup sci.techniques.xtallography is a great new tool for powder diffractionists.

Deane K. Smith Editor-in-Chief 